\title{
Resultados de la reanimación cardiopulmonar en pacientes pediátricos hospitalizados
}

\author{
José Baeza R.1; Verónica Gaete P.'; Ximena Cabellos M.2; Jaime Cordero T.1
}

\section{Cardiopulmonary resuscitation}

\begin{abstract}
Cardiopulmonary resuscilation is often done in hospital polienls with talher poor survival rales and outcome. This is a prospective descripfion on resuits of the procedure in 34 patienls Iram general pediatric wards -emesgency room and intensive care unil excluded-who develcped cardiopulmonary arrest. Resuscitation was done by previously aganized leams, whose personel was trained in accordance with the stondards of the American Hearl Association and the American Acodemy of Pediatrics. The mean age ol palients al cordiopulmonary arrest was 8 months irange 1 day to 14 years), 27/34 (79\%) had some chronic disease and the mean duralion of their hospital stoy wos nine days. Resuscitation maneuvers were starled within five minules in $82 \%$ 28/34) cases, all were mask ventilated. in $71 \%(24 / 34)$ patients inlubation was necesary and it was successfully achieved in $92 \%\{21 / 24\}$. More than one epinephrine dose was used in $6 \mathrm{~B} \%\{23,34\}$ coses. All drugs were delivered via peripheral veins in $79 \%[27 / 34\}$ patients. In six chidien in which vasculor access was not available al arrest it took more than 5 minules to start drug delivery, in five the endotraqueal roule wos used and an osteoclisis was employed in the remainder. The duration of arrest was 5 min or less in slx patients and longer than $30 \mathrm{~min}$ in 12 cases (38\%). Althrough initial success rale wos $53 \%$ (18/34j only o patjents were discharged from the hospital. No palient survived when cardiopulmonary arrest losted more thon 10 minules. Outcome of condiopulmonary resuscilotion is poor in pediatric inpatients with chronic diseases and probably "do not resuscitale" orders may be justified in many of them.
\end{abstract}

Key wordst hear arrest, cordiopulmonary resuscilalion, decision, outcome, do not resuscitale orders, infants, children.)

La reanimación cardiopulmonar $(\mathrm{RCP})$ es efectuada con frecuencia en pacientes hospitalizados. Su efectividad en estas condiciones no ha sido suficientemente validada ${ }^{1-3}$, existiendo consenso que depende de la causa del paro cardiorrespiratorio $(\mathrm{PCR})^{4,5}$, del estado de salud previo de los pacientes, del lugar donde ocurre $e^{6-9}$, de la prontitud y calidad de las técnicas de reanimación aplicadas por equipos entrenados y con roles previamente establecidos ${ }^{10,11}$.

Ampliando una investigación previa en pacientes de una unidad de cuidados intensivos ${ }^{12}$, diseñamos un estudio prospectivo destinado a describir las características de los pacientes que son reanimados en otras unidades del hospital, las causas del paro cardiorrespiratorio, la calidad

I. Unidad de Tratamiento Inlensivo, Hospital Luis Calvo Mackenna.

2. Enfermera Universitaria, Unidad de Laclantes, Hospital Luis Calvo Mackenns. y efectividad de las maniobras de resucitación efectuadas por equipos de residentes becarios previamente capacitados y organizados para cfectuar la reanimación y los resultados obtenidos en cl corto y mediano plazo en términos de sobrevivencia.

\section{Material y Método}

Durante un período de nucve meses se estudiaron en forna prospectiva los pacientes reanimados en las distintas unidades del hospital, excluidas emergencia y tratamiento intensivo. Antes de comenzar el estudio se formaron wcho equipos de reanimación, que cubrieran la atención de los unos. Cada equipo funcionó con un mínimo de tres becados residentes, una enferuera y Jos auxiliares paramédicos, cuyos roles se establecieron previamente (tabla 1). T'odos recibieron instrucción previa, teórica y práccica (maniquí de reanimación) por parte de los autores. Los equipos se activaron mediante scīales de radio emitidas por llamadores empleados con esta exciusiva finalidad. Se equipó a las diferentes unidades con carros de reaninación, docados con todo el material necesario para reanimación básica y avanzada. 


\section{Tabla 1}

Asignación de roles en тeanimación cardiopulmonar en pacientes hospitalizados

\begin{tabular}{|c|c|}
\hline Médico I & $\begin{array}{l}\text { (Residente } 3^{\text {es año) líder } 1} \\
\text { Dirige maniobras, valora efectividad y respuesta. Efectúa procedimientos de entubación, } \\
\text { instalación catéter central u osteoclisis. Indica medicamentos. Decide traslado o finalización } \\
\text { de los esfuerzos. Solicita asistencia a otros especialistas. }\end{array}$ \\
\hline Mếdico I] & : (Residente 2 año) \\
\hline Médico YII & $\begin{array}{l}\text { : (Residente let. año) } \\
: \text { Asume control de la circulación. Efectúa masaje cardíaco. }\end{array}$ \\
\hline Enfermera residente & $\begin{array}{l}\text { : Establece acceso vcnoso periférico. lnstala sonda nasogástrica. Infunde medicamentos. Toma } \\
\text { muestra exámenes. }\end{array}$ \\
\hline $\begin{array}{l}\text { A uxiliar } 1 \\
\text { A uxiliar } 2\end{array}$ & $\begin{array}{l}\text { : Prepara dilución estándar de medicamentos. Registra dosis de medicamentos. } \\
\text { : Aclúa como mensajero, iraslada exámenes o equipos requeritos durante la reanimación. }\end{array}$ \\
\hline
\end{tabular}

El diagnóstico de paro cardiorrespiralorio (paro) se hizo clínicamente, en ausencia de ruidos card́acos y pulso confirmadas por el lijer I $^{17}$. Se excluyeron los casos de paro respiratorio exclnsivo o bradicardia. En los pacientes en que se repitió el paro, sólo se consideró el primer evento. Los principios básicos y procedimientos de reanimación empleados fueron los establecidos por la Asociación Americana del Corazón y la Academia Amcricana de Pediatría, ambas de los Esiados Linidos 13, 14,

Inmedialamente despućs de concluida la reanimación, el jefe de cada equipo reunió los antecedentes en un registro tipo, anotando edad, sexo, tiempo de hospitalización, estado de salud previo, enfermedad actual y vigilancia del paciente, hora, día y causa del paro. Con respecto de la reanimación, se anotó demora en IJegar el equipo, lipo de veatilación empleada, entubación endoraqueal y número de intentos de conseguirla; vías vasculares de acceso, ticmpo invertido en establecerta; medicamentos empleados, dosis y, finalmente, resultados del procedimiento expresados en sobrevida y estado neurológico inmediato, 24 y 72 horas despućs de realizado aquét y al alta del paciente.

\section{Resultados}

Treinta y cuatro pacientes fueron reanimados durante el periodo de estudio, 14 eran mujeres. La media de edad del total fue 8 meses (márgenes 1 día a 14 afios). En 79,4\% (27/34) de los pacientes había una falla crónica de saludis, condicionada por enfermedades congénitas (dos anomalías cromosómicas, diez cardiopatías, una malformación del sistema nervioso central, dos (de otros tipos de malformaciones) o adquiridas (siete neumopatías, cinco enfermedades de la esfera oncológica, cuatro niños con retardo psicomotor). En ningún caso había en su ficha orden de no reanimar. La media de la duración de la hospitalización en el momento del paro era $9(\bar{x}$
$=28,9$ ) días, destacando que en once pacientes éste se produjo al cabo de un mes o más de permanecer en el hospital. No se encontró diferencia en la frecuencia con que el paro ocurrió, al comparar días festivos y laborales. El momento del suceso fue diumo $(8,00$ a $20,00 \mathrm{~h})$ en 14 , y nocturno en 20 pacientes y la afección aguda más relevante era respiratoria en 14 casos (41\%), infecciosa en $6(17,6 \%)$, oncológica en $6(17,6 \%)$, cardiaca en $5(14,7 \%)$ y neurológica en $3(8,8 \%)$. En $85 \%$ de los afectados había existido vigilancia médica aceptable y control en las seis horas precedentes. Las anormulidades fisiopatologicas principales que precedieron al paro fucron: hipoxia en 22 pacientes, cardíaca o circulatoria en seis, metabólica en dos, neurológica en uno y no precisable en tres.

El tiempo transcurrido entre la detección del paro y la llegada del equipo médico fue menor o igual a tres minutos en $82 \%(28 / 34)$ de los casos y en sólo uno mayor de 5 minutos. La ventilación inicial se hizo mediante ambu a la boca. En 24 niños en que ésta debió prolongarse se intentó entubación endotraqueal con $92 \%$ de buenos éxitos (22/24). En todos los afectados se realizó compresión cardíaca con técnica de masaje a tórax cerrado. Sólo un paciente respondio sin medjar medicamentos; en los restantes se empleó adrenalina (una dosis en ocho, dos o más dosis en 23), bicarbonato de sodio en 19 casos y atropina en 14 niños. La dosis inicial de adrenalina fue la convencional $(0,01 \mathrm{mg} / \mathrm{kg})$, pero en 22 pacientes se usaron, posteriormente, megadosis de $0,1 \mathrm{mg} /$ $\mathrm{kg}$. La administración de medicamentos se hizo por venas periféricas en $79,4 \%(27 / 34)$ de Jos 
casos. El tiempo que se tardó en obtcner esa vía fue más de 5 min en scis de ocho pacientes en que ella no habia sido preparada antes del paro. En cinco de ellos se emplé la ruta endotraqueal $y$ en uno osteoclisis.

Los resultados de la reanimación según la duración del paro se muestran en la labla 2. El paro se prolongó por 5 minutos o menos en seis pacientes y por más de 30 minutos en 12 . Aunque la sobrevida inmediata fue de $18 / 34(53 \%)$, sólo $10 / 34(29 \%)$ de estos niños sobrevivía a las 24 horas y $2 / 34(5,8 \%)$ al alta. Los dos sobrevivientes cursaban con afección pulmonar y cardíaca, respectivamente; respondieron a las maniobras antes de transcurrir 10 minutos ( 3 y 7 minutos) y no mostraban signos de déficit neurologico al egresar.

\section{Comentario}

Los resultados de la resucitación cardiorrespiratoria en niños han sido estudiados en casos extrahospitalarios ${ }^{8,9,16}$, en unidades de emergen$\mathrm{cia}^{7,17} \mathrm{y}$ de tratamiento intensivo ${ }^{12}$, pero existe poca información sobre su efectividad en salas de hospitalización convencional ${ }^{2,10}$. Estas suelen estar destinadas a atender pacientes agudos no críticos o crónicos, disponen de escasos o ningún método de vigilancia instrumental continuada, los enfermos son supervisados de manera intermitente (relación paciente-enfermcra 12:1; paciente-auxiliar 6:1), no cuentan con suficientes recursos para reanimación avanzada y la resucitación es, generalmente, asumida por personal no habituado a este lipo de práctica y sin la organización apropiada, lo que afecta negativa- mente los resultados. Conociendo esta situación, en nuestro estudio procedimos en primer lugar a preparar a los médicos residentes, enfermeras y auxiliares a cargo de la atención de esas unidades en técnicas de resucitación dando especial importancia a la formación de equipos con actividades preestablecidas, métodos de comunicación expeditos, registros óptimos de la atención y equipamiento adecuado con carros de reanimación.

La atención del paro fue oportuna en el tiempo que se tardó en iniciar las maniobras, manejo de vía aérca, uso y dosis de medicamentos, pero deficiente en obtener oportunamente el acceso vascular. Creemos que este ültimo rol, hasta ahora ejecutado por enfermeras debido a la falta de entrenamiento de los médicos, debe ser modificado - por lo menos para situaciones de emergencia- y constituir una responsabilidad compartida. La vía intraósea, de fácil acceso y pocas complicaciones, representa en este caso una buena alternativa ${ }^{18-20}$.

A pesar de la optimización de las maniobras de reanimación por parte de los residentes, los resultados en sobrevida fueron menores que lo esperado y lo obtenido en la unidad de cuidados intensivos del mismo hospital, en que la sobrevida al alta fue $16,9 \% \%^{12}$. Aunque es probable que ello se relacione con mayor precocidad en el reconocimiento del paro y comienzo de las maniobras en esta última, también debe influir el tipo de pacientes, pues en cuidados intensivos el procedimiento se aplica a los que sufren afecciones agudas y en forma selectiva. Este estudio muestra que en el resto del hospital la reanimación se usa sin distinción, principalmente en pacientes crónicamente enfermos, muchos de los cuales, a

Tabla 2

Sobrevida de 34 pacientes reanimados segút la duración del paro cardiorrespiratorio

\begin{tabular}{|c|c|c|c|c|c|c|}
\hline \multicolumn{2}{|c|}{$\begin{array}{l}\text { Duración paro } \\
\text { min }\end{array}$} & \multirow{2}{*}{$\frac{\begin{array}{c}\text { Pacientes } \\
\mathbf{n}\end{array}}{6}$} & \multirow{2}{*}{$\frac{\text { Inmediata }}{6}$} & \multirow{2}{*}{$\begin{array}{l}\text { Sobrevida } \\
\frac{24 \mathrm{~h}}{3}\end{array}$} & \multirow{2}{*}{$\frac{72 \mathrm{~h}}{3}$} & \multirow{2}{*}{$\frac{\text { Alta }}{1}$} \\
\hline$<$ & 5 & & & & & \\
\hline 6 & -10 & 4 & 4 & 2 & 2 & 1 \\
\hline 11 & -15 & 2 & 2 & 1 & 0 & 0 \\
\hline 16 & -30 & 10 & 4 & 3 & 2 & 0 \\
\hline$>$ & 30 & 12 & 2 & 1 & 1 & 0 \\
\hline Total & & 34 & 18 & 10 & 8 & 2 \\
\hline
\end{tabular}


pesar de su inestabilidad, no son trasladados a terapia intensiva, dadas sus escasas posibilidades de recuperación. La baja efectividad del procedimicnto en pacientes hospitalizados, especialmente con invalideces crónicas, descompensación cardiocirculatoria severa, fallas multiorgánicas, en ausencia de factores agudos desencadenantes, ha sido serialada en numerosos estutios y no ha mejorado en los últimos 20 años a pesar del entrenamicnto del personal y el mejoramiento técnico de los recursos. Como en muchos pacientes es, sin embargo, un procadimiento terapéutico válido, es preciso racionalizar su emplco. Los comités de ética pueden jugar un rol muy importante cn estc sentido, dirimiendo situaciones en que podría proceder la recomendación de no reanimar.

\section{Resumen}

Se estudiaron prospectivamente los resuliados de la reanimación cardiopulmonar efectuada en 34 pacientes internados en unidades convencionales de hospitalización (excluidos servicios de urgencias e intensivos), por cquipos previamente capacitados y organizados. Los pacientes reanimados en su mayoría fueron lactantes, portadores de falla crónica de salud (79\%) y al momento de la resucitación la media de duración de Ja hospitalización era 9 días. El equipo médico demoró 3 minutos 0 menos en iniciar las maniobras en $82 \%$ de los casos. Se intentó entubación traqueal en $71 \%$ de los pacientes, consiguiéndose en $92 \%$ de los intentos. En $91 \%$ de los afcetados fue necesario emplear medicamentos y $\mathrm{cn}$ $74 \%$ de éstos sc unilizó más đe una dosis de adrenalina. E1 acceso vascular en $79 \%$ fue una vena periférica y se tardó 5 minutos o más en oblenerse en 6 de 8 pacientes en que no cstaba disponible al ocurrír el paro. La duración del paro y de la reanimación fue 5 minutos o menos en $18 \%$ de los casos y más de 30 minutos en $38 \%$. Aunque el proccdimicnto fue inicialmentc efectivo en $53 \%$ de los intentos, sólo $5,8 \%$ de los pacientes sobrevivieron para scr dados de alta. Ningún paciente que requirió maniobras de reanimación por más de 10 minutos sobrevivió. La reanimación cardiorrespiratoria es poco efectiva en pacientes pediátricos hospitalizados, cspecialmente si se trata de enfermos crónicos, lo que sugiere la conveniencia de definir su em- pleo con mayor precisión y cventualmente considerar la orden de no rcanimar cuando sea pertinente.

(Palabras clave: resucilación cardiorrespiratoria, paro cardíaco, decisión de resucitar, órdenes de no resucitar.)

\section{Referencias}

1. Kvf J. Puri V, Raheja R, Ireland T: Cardiopulmonary resuscitation in hospilalized patients: continuing problems of decission-making. Crit Care Med 1987; $15: 41-43$.

2. Scon $R$ : Cardiopulmonary resuscitation in a reaching hospital. Anesthesia 1981; 36: $\$ 26$.

3. Bedell 5, Detbunco J, Cook E, et at.: Survival afier cardiopulmonary resuscitation in the hospital. N Fingl I Med 1983: 309: 569-576.

4. Nichols $D$, Kettrick $R$, Swedlow $D$, et al:: Factors influencing outcome of cardiopulmonary resuscrtation in children. Pedian Fmery Carc 1986; 2: 1-5.

5. Eisenberg M. Bergner L, Nallstrom A: Epidemiology of cardiac arrest and rcsuscilalion in children. Ann Emerg Med 1983; 11: 671-674

6. Ludwis $S$, Kettrick $R$, Parker M: Pediatric cardiopulmonary resuscitavion. A nevicw or 130 cases. Clin Pedialr 1984; 23: 71-75.

7. Rosenberg $N$ : Pediatric cardiopulmonary arrest in the emergency department. Am J Eroerg Med 1984; 2 : 497-501.

8. Thompson J, Bonner B, Lower O Pediatric cardiopulmonary arrest in rural nopulation. Pediatrics 1990; 86 : 302.306 .

9. Eisenberg M, Berger L, Hallstrom A: Paramedic programs of out of hospital cardiac artest: I Factors associated with succesfull resuscitation. Am I Public Ilcalth 1979; 69: 30-33.

10. Ehrlich $R$, Emmet $S$, Rodriguez-Torres $R$ : Pediatric cardiac resuscitation team: A 6 years study. J Pediatr $1974 ; 84: 152-155$

11. Gillis J, Dickson D, Rieder H. Steward D, Edmonds J: Results of inpatients pediatric resuscilation. Crit Care Med 1986; 14: 469.472

12. Baeza J, Ramírez K, Cordero J, et al.: Pano cardio. rrespiratorio. Resultados de la reanimación en UTT pediáırica. Rev Chit Pediát 1989; 60: 271-275.

13. Chamicles $L$ : Textbook of pediatric advanced life support. American Heart Associalion, Dallas, Tex., t990.

14. American Meart Association: Stundards and guidelines cardiopulmonary resuscitation and emergency cardiac Care. JAMA 1986; 255-2954-2960.

15. Baeza J, Miranda P, Rui Pérez E, Cordero $J_{\text {: Letalidad }}$ en unidad de [ratamiento intensivo pediátrica. [i] rol de la falla aguda de órganos y sistemas. Rey Chil Pcdiatr 1989; 60: 319-326.

16. O'Rourke P: Out-of hospital cardiac arrest in pediatric patients: oulcomc. Crit Care Mcd 1984; 12: 283-285.

17. Torphy DE, Minter MG. Thompson BH: Cardiorespiratory ames and tesucitation of children. Am J Dis Chitd 1984; 138: 1099-1102. 
18. Rosetti $V$, Thompson $B$, Mitter $J$, et al.: Intraosseous infusions. An altemative route of pediatric intravascular access. Ann Emerg Med 1985; 14: 885.888.

19. Sivey W: Intraosseous infusions. J Pediatr 1987; 111: 639-643.

20. Fiber D: Intraosseous infusions. N Engl J Med 1990; 322: $1579-1581$.
21. Davies $S M$, Reynold's BX: The ethics of cardjopuimonary resuscitation II medical logistics and the potential for good response. Arch Dis Child 1992; 67: 257-261.

22. Lantos J, Miles $S$, Silverstein M. Stocking C: Survival after cardiopulmonary resuscilation in babics of very low birth weight. Is CPR fulile therapy? N Engl I Med 1988; 318: 91-95. 